Short communication

\title{
Use of the nutritional risk score by surgeons and nutritionists
}

\author{
Michael Benoit ${ }^{a}$, Fabian Grass ${ }^{a}$, Nicolas Demartines ${ }^{a},{ }^{*}$, Pauline Coti-Bertrand ${ }^{\text {b }}$, \\ Markus Schäfer ${ }^{a}$, Martin Hübner ${ }^{a}$ \\ a Department of Visceral Surgery, University Hospital CHUV, Lausanne, Switzerland \\ ${ }^{\mathrm{b}}$ Department of Endocrinolgy, Diabetology and Metabolism, Unit of Clinical Nutrition, University Hospital CHUV, Lausanne, Switzerland
}

\section{A R T I C L E I N F O}

\section{Article history:}

Received 10 August 2014

Accepted 21 January 2015

\section{Keywords:}

Nutritional screening

Nutritional risk score

Perioperative nutrition

Reliability

\begin{abstract}
S U M M A R Y
Background: The Nutritional Risk Score (NRS) is a validated tool to identify patients who should benefit of nutritional interventions. Nutritional screening however has not yet been widely adopted by surgeons. Furthermore, the question about reliability of nutritional assessment performed by surgeons is still unanswered.

Methods: Data was obtained from a recent randomised trial including 146 patients with an NRS $\geq 3$ as assessed by the surgeons. Additional detailed nutritional assessment was performed for all patients by nutritional specialists and entered prospectively in a dedicated database. In this retrospective, surgeons' scoring of NRS and its components was compared to the assessment by nutritionists (considered as gold standard).

Results: Prospective NRS scores by surgeons and nutritionists were available for 141 patients (97\%). Surgeons calculated a NRS of 7, 6, 5, 4 and 3 in 2, 8, 38, 21 and 72 patients respectively. Nutritionists calculated a NRS of 6, 5, 4, 3 and 2 in 8, 26, 47, 57, 3 patients, respectively. Surgeons' assessment was entirely correct in 56 patients (40\%), while at least the final score was consistent in 63 patients (45\%). Surgeons overrated the NRS in $21 \%$ of patients and underestimated the score in $29 \%$. Evaluation of the nutritional status showed most of the discrepancies (54\%).

Conclusion: Surgeon's assessment of nutritional status is modest at best. Close collaboration with nutritional specialists should be recommended in order to avoid misdiagnosis and under-treatment of patients at nutritional risk.
\end{abstract}

๑) 2015 Elsevier Ltd and European Society for Clinical Nutrition and Metabolism. All rights reserved.

\section{Introduction}

Despite the fact that malnutrition is nowadays accepted as an important risk factor that impacts on postoperative morbidity and infectious complications in particular; accurate assessment is still a matter of debate [1-4]. Nevertheless there is solid evidence in the literature that perioperative nutritional interventions are highly effective and guidelines provided by American and European Nutritional Societies are available for a standardised assessment and tailoring nutritional interventions [5-8].

Aiming to identify patients at risk, the Nutritional Risk Score (NRS) has been developed as a prospectively validated screening tool in Europe [9]. Based on few items which are easy to obtain, a reliable identification of malnourished patients should be possible, independently whether users are specialists in clinical nutrition or not.

In general, preoperative nutritional assessment of surgical patients is performed either by surgeons, nurses, or by nutrition specialists [4]. However, there is little information available, whether the application of the NRS is really easy and reproducible in daily clinical practice, and how important the inter-observer variability is between surgeons and nutrition specialists.

The aim of the current study was to evaluate eventual differences in NRS scoring performed by surgeons or nutrition specialists prior to major abdominal surgery.

\footnotetext{
Abbreviations: NRS, nutritional risk score.

* Corresponding author. Department of Visceral Surgery, CHUV - Centre Hospitalier Universitaire Vaudois, Rue du Bugnon 46, 1011 Lausanne, Switzerland. Tel.: +412131424 00; fax: +41213142411.

E-mail address: demartines@chuv.ch (N. Demartines).
}

\section{Methods}

This is a retrospective analysis of a prospective database from a recently published trial (NCT00512213) [10]. In a double-blinded 
randomised trial, 146 patients undergoing major abdominal surgery were randomised to receive either immunonutrition ${ }^{\circledR}$ or an isocaloric-isonitrogenous oral solution during the last five days prior to surgery. Only patients at nutritional risk as defined by a NRS score $\geq 3$ were eligible for this study.

Initial nutritional screening was performed by the surgical team (staff surgeons and residents) in the outpatients' clinic using the NRS-score. All eligible patients were informed about the study and included in the protocol once their written consent was obtained. All study participants were then assessed by the nutritional team (nutrition specialists and nurses) who realised a broad nutritional assessment including NRS. This was a unique opportunity to scrutinize a well-documented cohort of consecutive patients with prospectively performed NRS scoring by surgeons and by nutritionists.

The NRS is a multimodal screening tool in which disease severity, nutritional status and age are combined [9]. Nutritional status is evaluated by three variables: Body Mass Index (BMI), recent weight loss and food intake during the last week before evaluation. Severity of disease, used as an indicator of metabolic stress and increased nutritional requirements, is graded on a scale from 1 to 3 [9]. One point is added for patients aged $>70$ years. A NRS-score of $>3$ is considered as at risk [9]. The surgical team had repetitive educational sessions where the NRS was explained in detail; practical exercises were performed to reinforce the message.

For the purpose of this study, we defined the nutritionists' assessment as gold standard and as reference for the surgeons' evaluation. Descriptive statistics were prepared and presented using Numbers 3.2 (Apple Inc., 1 Infinite Loop, Cupertino, CA 95014 USA). Categorical variables were tested for statistical significance $(P<0.05)$ applying Chi square test by use of Prism 5.2 (GraphPad ${ }^{\circledR}$ Software Inc., 2236 Avenida de la Playa, La Jolla, CA 92037 USA). Inter-rater agreement test (kappa test) was used to assess agreement between examiners. We used linear weights since the difference between the first and second category has the same importance as a difference between the second and third category (MedCalc Software, Version 12.4.0; B-8400 Ostend, Belgium).

\section{Results}

Prospective NRS scores were assessed independently by surgeons and nutritionists for 141 out of 146 patients (97\%). The global scoring of the NRS by surgeons and nutritionists is displayed in Table 1. Surgeons calculated a NRS of 7, 6, 5, 4 and 3 in 2, 8, 38, 21 and 72 patients respectively. Nutritionists calculated a NRS of 6,5 , 4,3 and 2 in 8,26, 47, 57, 3 patients, respectively. The surgeons' estimates arrived at the same final score in 63 patients (45\%), but were entirely consistent with nutritional assessment in 56 patients (40\%) only $(P=0.002)$. Surgeons overestimated the NRS final score

Table 1

Nutritional assessment by surgeons versus nutritionists.

\begin{tabular}{lll}
\hline NRS score & Surgeon & Nutritionist \\
\hline 2 & $0(0 \%)$ & $3(2 \%)$ \\
3 & $72(51 \%)$ & $57(40 \%)$ \\
4 & $21(14 \%)$ & $47(33 \%)$ \\
5 & $38(27 \%)$ & $26(18 \%)$ \\
6 & $8(6 \%)$ & $8(5 \%)$ \\
7 & $2(1 \%)$ & $0(0 \%)$ \\
\hline
\end{tabular}

Overall NRS scores attributed by surgeons and nutritionist for the same cohort of 141 patients.

NRS - Nutritional Risk Score.
125

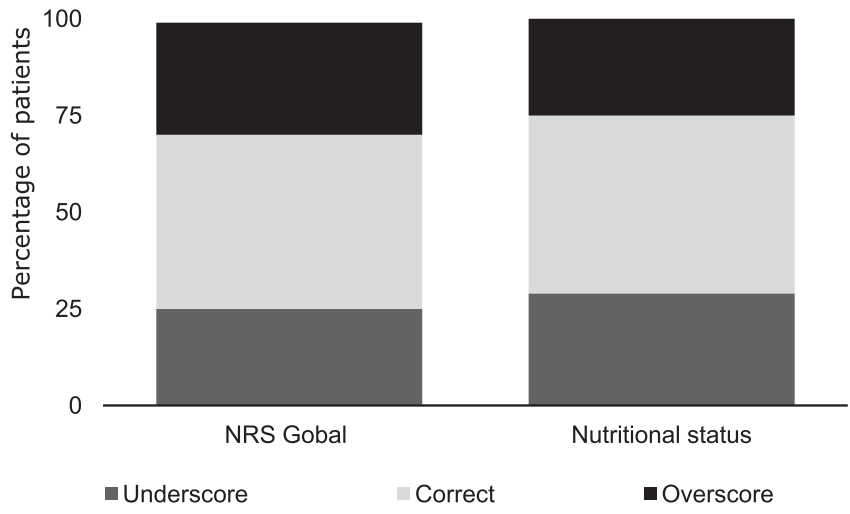

Fig. 1. Scoring quality of surgeons compared to nutritionists. NRS scoring performed by the surgical team was compared with the assessment done by the nutritional specialists. The number of congruent ratings is displayed in grey, whereas higher and lower attributed scores as visualized in black and white, respectively, for NRS overall on the left and its component nutritional status on the right side. NRS - Nutritional Risk Score.

in $21 \%$ of patients and underscored in $29 \%$ (Fig. 1). A detailed overview of discrepancies between surgical and nutritional assessment is provided in Table 2 .

The main difference in nutritional screening performed by surgeons and nutritionists regarded the nutritional criteria of the NRS with incongruent assessment in 77 patients representing 55\% of the cohort $(P<0.001)$. Of these, 42 patients $(29 \%)$ were attributed a lower scoring for their nutritional status by surgeons, while 35 patients (26\%) were overscored by the surgeons' assessment as compared with the evaluation by the nutritional team (Fig. 1).

There were also differences noted between surgeons and nutritionists in assessing disease severity. Discrepant scoring occurred in overall 17 patients (12\%); in 16 patients surgeons attributed a higher score than nutritionists, while the opposite was observed in one patient only $(P=0.005)$. Unfortunately, one surgeons forgot by mistake to add an extra-point for old age, while the respective patients was exactly 70 years.

A weighted Kappa value was calculated to evaluate inter-rater agreement (see Table 3). We calculated a value of 0.303 (95\% CI: 0.184-0.423), which interpreted with Altman's index is evaluated as fair.

Table 2

Assessment of the components of NRS by surgeons and nutritionists.

\begin{tabular}{lccc}
\hline NRS & Score & Nutritionist $N=141$ & Surgeon N = 141 \\
\hline Nutritional status & 0 & 40 & 44 \\
& 1 & 40 & 38 \\
& 2 & 42 & 38 \\
Disease severity & 3 & 19 & 21 \\
& 0 & 2 & 0 \\
& 1 & 3 & 0 \\
Age $>70$ & 2 & 135 & 130 \\
& 3 & 1 & 11 \\
& 0 & 62 & 62 \\
& 1 & 79 & 79
\end{tabular}

The components of the NRS as documented by surgeons and nutritionist for the same cohort of 141 patients.

NRS - Nutritional Risk Score. 
Table 3

Inter-rater agreement test (Kappa test).

\begin{tabular}{|c|c|c|c|c|c|c|c|}
\hline \multirow[b]{2}{*}{ Observer B } & \multicolumn{6}{|c|}{ Observer A } & \\
\hline & 1 & 2 & 3 & 4 & 5 & 6 & \\
\hline 1 & 0 & 0 & 0 & 0 & 0 & 0 & $0(0.0 \%)$ \\
\hline 2 & 0 & 0 & 0 & 0 & 0 & 0 & $0(0.0 \%)$ \\
\hline 3 & 1 & 2 & 38 & 21 & 6 & 3 & $71(50.4 \%)$ \\
\hline 4 & 0 & 1 & 7 & 12 & 4 & 0 & $24(17.0 \%)$ \\
\hline 5 & 0 & 3 & 9 & 12 & 10 & 2 & $36(25.5 \%)$ \\
\hline \multirow[t]{2}{*}{6} & 0 & 0 & 0 & 0 & 7 & 3 & $10(7.1 \%)$ \\
\hline & $1(0.7 \%)$ & $6(4.3 \%)$ & $54(38.3 \%)$ & 45 (31.9\%) & 27 (19.1\%) & $8(5.7 \%)$ & 141 \\
\hline \multicolumn{3}{|c|}{ Weighted Kappa ${ }^{a}$} & & & & & 0.303 \\
\hline \multicolumn{3}{|c|}{ Standard error } & & & & & 0.061 \\
\hline \multicolumn{3}{|c|}{$95 \% \mathrm{CI}$} & & & & & 0.184 to 0.423 \\
\hline
\end{tabular}

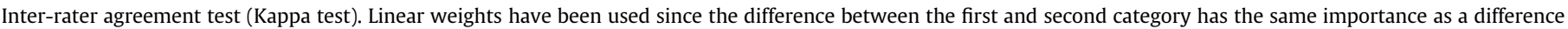
between the second and third category, etc.

a Some explanation to the statistical methods used.

\section{Discussion}

The surgeons' assessment of the nutritional status by use of the NRS-2002 is in our experience modest at best despite comprehensive and repetitive education. Overall, surgeons tended to underscore the nutritional status and to overrate the disease severity as compared with evaluation by nutritional specialists. The NRS is apparently not as easy to use as initially suggested and sources of errors are found at various levels.

Nutritional screening is an important first step in order to identify patients who need to have nutritional interventions in order to reduce (infectious) complications and hospital stay $[5,7,11-13]$. In reality, about $30 \%$ of hospitals rarely or never assess patients' nutritional status preoperatively as recently published by our group [4]. Our findings are in accordance with a recent European survey showing that only $52 \%$ of the interrogated units reported a screening routine [14]. This has to be considered as an important shortcoming in modern GI surgery [15]. Furthermore, $48 \%$ of the centers evaluated their patients' nutritional status at admission only or even postoperatively, which is obviously too late for an effective nutritional intervention [4].

The nutritional risk score (NRS) is officially recommend as screening tool since 2003 [7]. The NRS is a validated tool proclaimed to be fast and easy to use - both mandatory prerequisites for surgeons. The group of Jie et al. [16] showed that preoperative nutritional support was beneficial to patients with an NRS-2002 score of 5 or more by lowering complication rates. However, the use of the NRS remains quite limited even in European countries $[4,14]$. Time restrain has been identified as one explanation for not using the NRS-2002 screening tool [4], but this is probably not the entire truth. Another reason might be its relative (unapparent) complexity. Although claimed to be simple and straightforward, several components of the score are ill-defined and/or time consuming. And indeed, our analysis pointed clearly out that surgeons, although well aware about the score and its importance, were evidently not able to assess the score correctly in more than half of the patients. Explanations are multifold. On the one hand, nutritional status is evaluated by three different variables: body mass index (BMI), recent weight loss and food intake during the last week before evaluation. The latter two parameters are highly subjective and not always easy to define in elder and frail patients. To amalgam the obtained information into one single score for nutritional status might not always be self-evident in a busy surgical outpatient consultation where nutritional screening is just one out of many other very important points.

The inter-observer variability was confirmed by use of an interrater agreement test (Kappa test). We calculated a weighted kappa value of 0.303 . This result can be interpreted as a fair strength of agreement between observers by Altman. Hence, we can confirm that there is an observer difference between surgeons and nutritionists when evaluating the same patients using the same evaluation tool.

Therefore, precise assessment of weight loss and food intake is better done by experienced nutritionists than surgeons in training. Moreover, to correctly assess weight loss prerequisites that patients have a weighing scale at home. Furthermore, especially male patients are not used to regularly assess their body weight.

We believe the NRS-Score is a complex tool to use even for trained nutritionists. Especially the evaluation of nutrition intake during one week in reference to the patients weekly requirements is quite a task. Already for a trained nutritionist, so how much more for a quickly trained surgeon.

On the other hand, disease severity or the magnitude of a planned surgical intervention is not clearly defined. The important discrepancies in scoring between nutritionists and surgeons in our study underline this problem. It remains questionable whether nutritionists are able to apprehend the tremendous differences between different types of surgeries. This hypothesis is confirmed by the fact that nutritionists gave almost exclusively a score of 2 for this item. Standardized lists of interventions are certainly misleading, as the mere name of a procedure does not automatically reflect the magnitude of surgical stress. A traditional open colectomy will induce a very different stress response compared with a laparoscopic colon resection within a multimodal enhanced recovery pathway.

Given the complexity of the NRS-score and the fact that it is not easy to use in a daily practice, alternative evaluation tools should be provided to clinicians. This is confirmed by Schindler et al. [14] who showed that nutritional routines and nutritional care remain poor in Europe possibly because of lack of an easy to use evaluation tool. One potential alternative to the NRS-score is the Malnutrition Universal Screening Tool (MUST) developed by the British association for parenteral and enteral nutrition (BAPEN). Even though the MUST Score has been clinically validated [17] we are not aware of any study comparing the MUST to the NRS-score.

Several limitations need to be addressed. This is a retrospective analysis and the study cohort is limited. The data however was retrieved from a well documented prospective database and was almost complete. Synchronous but independent nutritional screening comparing the performance of surgeons vs. nutritionists has not been performed to the best of our knowledge. Nutritionists' assessment was here considered as the gold standard. Although, specialists might obviously do wrong, a retrospective assessment of the "true NRS" appeared to be unrealistic and prone to bias. In 
addition, our study included only patients scored NRS $\geq 3$. The interesting question of how many patients at nutritional risk have been underscored by surgical screening and thus elapsed nutritional intervention could therefore not been answered. The $29 \%$ of underscored patients in our study cohort provide a rough estimate but future investigations need to confirm these findings.

In conclusion, surgeons' assessment of nutritional status - if performed at all - appears not to be entirely reliable. The NRS seems to be more difficult to use as suggested. Not only the assessment of nutritional status is difficult, but also the grading of disease severity are sources of error leading to an inaccurate scoring in about half of the patients. The surgeons' notorious lack of time but also their limited expertise point out the importance of a multidisciplinary team approach integrating the correct evaluation of the patients' nutritional condition.

\section{Conflict of interest}

The authors declare no conflict of interest.

\section{Acknowledgements}

The authors thank Giustina Mariotti for her valuable help in data acquisition and management.

\section{References}

[1] McWhirter JP, Pennington CR. Incidence and recognition of malnutrition in hospital. BMJ 1994;308:945-8.

[2] Waitzberg DL, Saito H, Plank LD, Jamieson GG, Jagannath P, Hwang TL, et al Postsurgical infections are reduced with specialized nutrition support. World J Surg 2006;30:1592-604.

[3] Schwegler I, von Holzen A, Gutzwiller JP, Schlumpf R, Muhlebach S, Stanga Z. Nutritional risk is a clinical predictor of postoperative mortality and morbidity in surgery for colorectal cancer. Br J Surg 2010;97:92-7.
[4] Grass F, Cerantola Y, Schafer M, Muller S, Demartines N, Hubner M. Perioperative nutrition is still a surgical orphan: results of a Swiss-Austrian survey. Eur J Clin Nutr 2011;65:642-7.

[5] Kondrup J. ESPEN guidelines for nutrition screening 2002. Clin Nutr 2003;22: $415-21$.

[6] Jones NEHD. Pharmaconutrition: a new emerging paradigm. Curr Opin Gastroenterol 2008;24:215-22.

[7] Sorensen J, Kondrup J, Prokopowicz J, Schiesser M, Krahenbuhl L, Meier R, et al. EuroOOPS: an international, multicentre study to implement nutritional risk screening and evaluate clinical outcome. Clin Nutr 2008;27:340-9.

[8] American Society for Parenteral Enteral Nutrition Board of D. Clinical guidelines for the use of parenteral and enteral nutrition in adult and pediatric patients. JPEN J Parenter Enteral Nutr 2009;2009(33):255-9.

[9] Kondrup J. Nutritional risk screening (NRS 2002): a new method based on an analysis of controlled clinical trials. Clin Nutr 2003;22:321-36.

[10] Hubner M, Cerantola Y, Grass F, Bertrand PC, Schafer M, Demartines N. Preoperative immunonutrition in patients at nutritional risk: results of a doubleblinded randomized clinical trial. Eur J Clin Nutr 2012;66:850-5.

[11] Weimann A, Braga M, Harsanyi L, Laviano A, Ljungqvist O, Soeters P, et al. ESPEN Guidelines on Enteral Nutrition: surgery including organ transplantation. Clin Nutr 2006;25:224-44.

[12] Cerantola Y, Hubner M, Grass F, Demartines N, Schafer M. Immunonutrition in gastrointestinal surgery. Br J Surg 2011;98:37-48.

[13] Weimann A, Breitenstein S, Breuer JP, Gabor SE, Holland-Cunz S, Kemen M, et al. Clinical nutrition in surgery. Guidelines of the German Society for Nutritional Medicine. Der Chirurg Zeitschrift fur alle Gebiete der operativen Medizen 2014;85:320-6.

[14] Schindler K, Pernicka E, Laviano A, Howard P, Schutz T, Bauer P, et al. How nutritional risk is assessed and managed in European hospitals: a survey of 21,007 patients findings from the 2007-2008 cross-sectional nutritionDay survey. Clin Nutr 2010;29:552-9.

[15] Bozzetti F, Gianotti L, Braga M, Di Carlo V, Mariani L. Postoperative complications in gastrointestinal cancer patients: the joint role of the nutritional status and the nutritional support. Clin Nutr 2007;26:698-709.

[16] Jie B, Jiang ZM, Nolan MT, Zhu SN, Yu K, Kondrup J. Impact of preoperative nutritional support on clinical outcome in abdominal surgical patients at nutritional risk. Nutrition 2012;28:1022-7.

[17] Stratton RJ, Hackston A, Longmore D, Dixon R, Price S, Stroud M, et al Malnutrition in hospital outpatients and inpatients: prevalence, concurrent validity and ease of use of the 'malnutrition universal screening tool' ('MUST') for adults. Br J Nutr 2007;92:799. 